\title{
QUIMIOPREVENÇÃO DO CÂNCER DE MAMA
}

\author{
Vilmar Marques de Oliveira*, José Mendes Aldrighi, José Francisco Rinaldi
}

Trabalho realizado no Departamento de Obstetrícia e Ginecologia da Faculdade de Ciências Médicas da Santa Casa de SP e Departamento de Saúde Materno-Infantil da Faculdade de Saúde Pública da USP, São Paulo, SP

*Correspondência:

vilmarmarques@uol.com.br

\section{RESUMO}

Quimioprevenção é definida como o uso de agentes químicos naturais ou sintéticos para reverter, suprimir ou prevenir a progressão carcinogênica para carcinoma invasor. Os fármacos que agem como agentes quimiopreventivos contra o câncer de mama são divididos em dois grupos principais: os que previnem cânceres de mama receptor de estrogênio (RE) positivos, como os moduladores seletivos do receptor de estrogênio (SERM), inibidores de aromatase, agonistas de GnRH e fitoestrogênios; e os fármacos que previnem os cânceres RE-negativos, como os inibidores da ciclooxigenase2 (COX-2), retinóides, as estatinas, os inibidores do receptor tirosina quinase, o anticorpo monoclonal contra HER-2 e os inbidores da telomerase. Resultados do estudo conduzido pelo NSABP que comparou o tamoxifeno com o raloxifeno (STAR), avaliando a eficácia na redução de risco, assim como a toxicidade desses dois SERMs em uma população similar e de alto risco para câncer de mama, demonstrou que o raloxifeno é tão efetivo quanto o tamoxifeno na redução de risco de câncer de mama invasor $(p=0,83)$ e apresentou menor risco de eventos tromboembólicos e catarata; todavia, exibiu maior risco de carcinoma não invasor, porém sem significância estatística. Baseado nos dados promissores que revelaram diminuição de risco de câncer de mama contralateral em estudos de adjuvância, alguns inibidores de aromatase, incluindo o letrozol, anastrazol e exemestane, estão sendo incorporados em investigaç̧oses para avaliar sua eficácia como agentes preventivos de alto risco em mulheres. Os inibidores de COX-2 demonstraram sua eficácia na prevenção do câncer de mama em estudos caso-controle e coorte, sendo necessários estudos aleatórios para atestar sua eficácia. O resultado positivo de alguns ensaios clínicos na prevenção do câncer de mama em populações de alto risco sugere que a quimioprevenção é uma estratégia racional e atraente.

UnIteRMos: Câncer de mama. Quimioprevenção. Tamoxifeno. Raloxifeno. Aromatase. Ciclooxigenase-2.

\section{INTRODUÇÃo}

Nas últimas décadas ocorreu expressivo aumento da incidência e mortalidade do câncer de mama em todo o mundo. Ao que tudo indica, esta neoplasia maligna é o resultado da interação de fatores genéticos com estilo de vida, hábitos reprodutivos e meio ambiente!.

Nos Estados Unidos, estima-se que uma em oito mulheres que atingiram os 80 anos irá desenvolver câncer de mama². No Brasil, excluindo-se os cânceres de pele, o câncer de mama representa a neoplasia maligna de maior incidência e mortalidade que acomete 0 sexo feminino. Para o ano de 2006, estima-se o aparecimento de 48.930 novos casos da neoplasia em todo o Brasil, com taxa bruta de 52,93 por 100.000 mulheres $^{3}$.

Todos os cânceres mamários têm origem gênica. Acredita-se que 90\% a 95\% deles sejam esporádicos (não familiares) e decorram de mutações somáticas, que se verificam durante a vida, e que $5 \%$ a $10 \%$ sejam hereditários (familiares), devido a mutações nucleotídicas perpetuadas na linhagem familiar pelas células germinativas, que confere suscetibilidade ao câncer de mama4.

Ainda que o processo carcinogênico deva ser conduzido por mutações gênicas, existem, claramente, muitas variáveis epigenéticas, particularmente aquelas relacionadas com ações autócrinas, parácrinas e endócrinas de moléculas reguladoras. As modulações farmacológicas destas vias reguladoras oferecem grande potencial para a prevenção do câncer ${ }^{5}$.
A despeito do diagnóstico precoce e dos avanços no tratamento do câncer de mama, a morbidade e a mortalidade associadas a esta doença permanecem elevadas ${ }^{6}$. A prevenção primária, portanto, parece oferecer as melhores chances de impacto favorável sobre esta neoplasia; a mesma está embasada na quimioprevenção, estilo de vida e nas cirurgias de redução de risco (mastectomias, adenectomias e ooforectomias bilaterais $)^{7}$.

Quimioprevenção é definida como o uso sistêmico de agentes químicos naturais ou sintéticos para reverter ou suprimir a passagem de lesões pré-malignas para carcinomas invasores ${ }^{8}$. Entre esses se destacam os moduladores seletivos de receptor de estrogênio (SERM), os inibidores da aromatase, os fitoestrogênios e os agonistas de GnRH na prevenção dos tumores mamários receptor de estrogênio (RE) positivos; e os inibidores da ciclooxigenase-2 (COX-2), retinóides, estatinas, inibidores do receptor de tirosina quinase (gefitinibe), anticorpo monoclonal contra HER-2 (trastuzumab) e os inibidores da telomerase, nos tumores RE negativos?.

\section{Tamoxifeno}

O tamoxifeno é um SERM que apresenta ação antiestrogênica na mama por se ligar ao RE e impedir, de forma competitiva, a ação do estrogênio no tecido mamário, além de agir sobre os fatores de crescimento TGF-a, TGF-b e IGF-I, propi- 
ciando, também, o aumento da SHBG (globulina carreadora de hormônios sexuais), com conseqüente diminuição dos estrogênios livres.

A primeira observação clínica da eficácia do tamoxifeno como agente preventivo do câncer de mama foi relatada por Cuzick \& Baum, em 1985, mostrando o decréscimo na incidência do câncer de mama contralateral em usuárias de tamoxifeno ${ }^{9}$. Estes achados foram ratificados pelos resultados do estudo NSABP B- $\mid 4^{10}$, em que se observou redução de 37\% na incidência de câncer de mama na mama contralateral em usuárias de tamoxifeno por cinco anos, após dez anos de estudo. Estes resultados respaldaram a elaboração de ensaios clínicos para balizar o uso do tamoxifeno na prevenção do câncer de mama.

As maiores evidências da eficácia da prevenção primária vêm dos ensaios clínicos aleatórios, que utilizaram como modulador seletivo de receptor de estrogênio o tamoxifeno ${ }^{11,12,13,14}$.

Em estudo conduzido pelo National Surgical Adjuvant Breast and Bowel project (NSABP-PI)"', que envolveu I3.388 mulheres, estas com idade igual ou superior aos 60 anos independente do risco de câncer de mama, e igual ou superior a 35 anos e inferior a 60 anos, apresentando risco preditivo de câncer de mama em cinco anos igual ou superior a I,66\%, segundo o modelo de risco proposto por Gaill ${ }^{5}$ (presença de mutações gênicas no gene BRCA- I ou BRCA-2, dois ou mais parentes em primeiro grau com carcinoma de mama na pré-menopausa, biópsia prévia revelando hiperplasia atípica ou carcinoma lobular in situ ou carcinoma de mama prévio), observaram no grupo tratado com tamoxifeno 20 mg redução de 49\% no risco de câncer invasivo. A diminuição do risco ocorreu em todas as faixas etárias e foi de $44 \%$ em mulheres com 49 anos ou menos, $51 \%$ naquelas com idade entre 50 e 59 anos e 55\% nas mulheres com 60 anos ou mais. O fármaco também reduziu o risco de câncer não invasivo em 50\%. Particularmente, foi importante a observação que o tamoxifeno reduziu em 69\% a incidência de câncer invasivo com RE-positivo, entretanto não houve diferença na incidência do câncer invasivo com RE-negativo entre os grupos placebo e tamoxifeno.

O estudo NSABP_PI teve seu seguimento estendido para sete anos, após o comunicado inicial dos resultados. Concluído esse período, foi constatado que o efeito preventivo apresentou pequena queda (49\% contra 43\%), sendo que a diferença não se mostrou estatisticamente significante ${ }^{16}$.

Não obstante os resultados do NSABP_PI, outros dois estudos, um inglês (Royal Marsden Trial - RMT) e outro italiano (Italian National Trial - INT), de tamanhos amostrais menores que o norte-americano e características populacionais diferentes, não apresentaram os mesmos resultados quanto à prevenção; utilizando-se de intervalo de confiança (IC) de $95 \%$, observaram os seguintes riscos relativos (RR) respectivamente: 0,83 (IC: 0,58-I, I6) e 0,76 (IC: 0,47-I, I6) 12, I3.

No estudo IBIS-I (International Breast Cancer Interventional Study) ${ }^{14}, 7.139$ mulheres foram randomizadas para receber placebo ou $20 \mathrm{mg} /$ dia de tamoxifeno, sendo elegíveis aquelas entre 35 e 70 anos de idade e que apresentavam risco relativo para câncer de mama aumentado em dez vezes para aquelas com idade entre 35-39 anos, quatro vezes quando entre 40-44 anos e de duas vezes para as que estavam entre os 45-75 anos. Após
Figura I - Risco relativo para todos os tipos de câncer de mama nos diversos estudos e na metanálise conduzida por Cuzick et al., 2003

Todos os casos de câncer (incluindo CDIS)

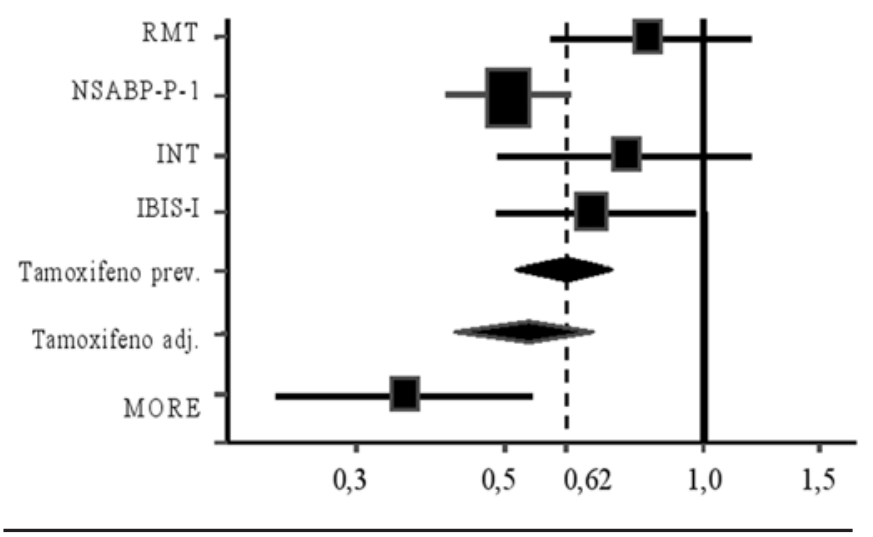

Figura 2 - Risco relativo para os fenômenos tromboembólicos nos diversos estudos e na metanálise conduzida por Cuzick et al., 2003

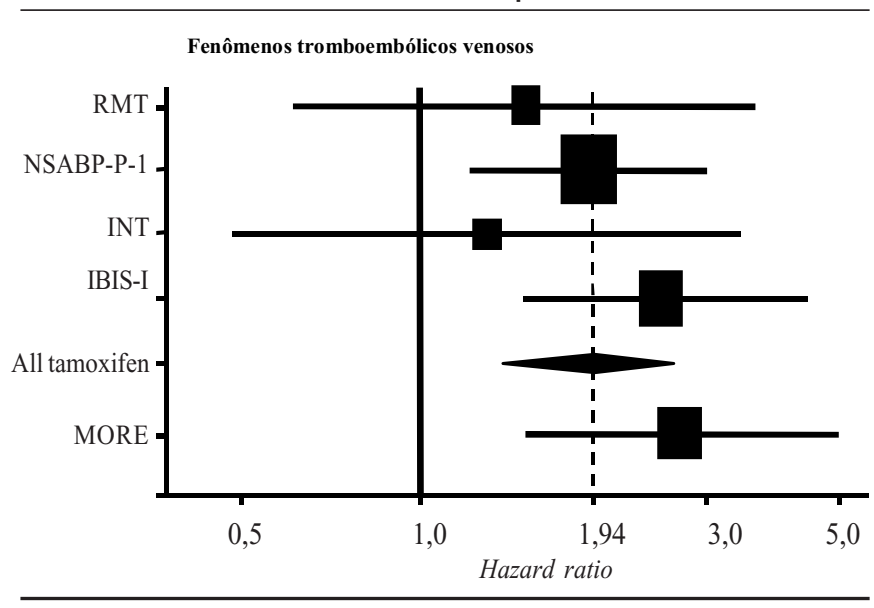

50 meses, 170 casos de câncer de mama foram reportados, sendo I0 I casos no grupo placebo e 69 no grupo tratado com tamoxifeno, traduzindo uma redução de $32 \%$ na incidência do câncer mamário $(p=0,013)$.

Em estudo metanalítico ${ }^{17}$, em que foram analisados os estudos NSABP-PI, RMT, INT, IBIS-I e MORE, assim como aqueles que usaram o tamoxifeno na adjuvância (NSABP-BI 4 e B24), constatou-se redução de $38 \%$ na incidência de câncer de mama nas pacientes usuárias de tamoxifeno. Os resultados estão sumarizados na Figura I, e os principais efeitos adversos (fenômenos tromboembólicos e câncer de endométrio) nas Figuras 2 e 3, respectivamente. Fica claro, observando estes resultados, que o uso do raloxifeno não acarreta risco para o desenvolvimento do câncer de endométrio, mas determina maior risco para tromboembolismo, ainda que não estatisticamente significante, quando em comparação com os outros estudos. 


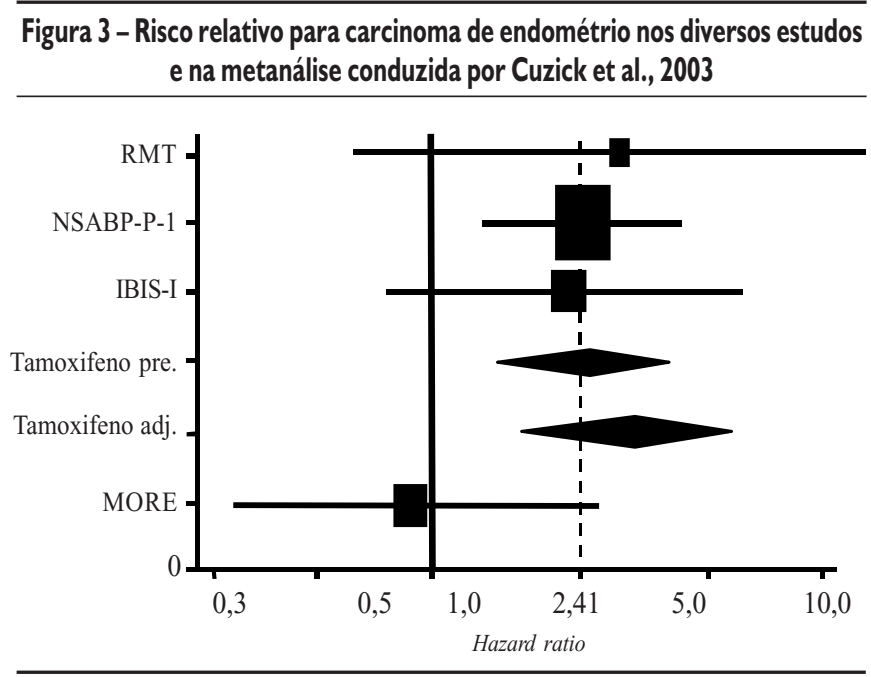

\section{Raloxifeno}

O raloxifeno é um SERM com comportamento clínico e biológico distinto do tamoxifeno; de fato, apresenta como diferença marcante o não estímulo endometrial, apesar de exercer as mesmas propriedades benéficas do tamoxifeno sobre o tecido mamário. Em estudos pré-clínicos, o raloxifeno mostrou prevenir o surgimento de novos cânceres mamários, assim como impedir o crescimento de cânceres pré-existentes quando da sua administração ${ }^{18}$.

Dois ensaios clínicos, para testar a eficácia do raloxifeno na prevenção da osteoporose, identificaram que este poderia reduzir a incidência do câncer de mama. O estudo MORE (Multiple Outcomes of Raloxifen Evaluation), randomizou 7.704 pacientes pós-menopáusicas, com média etária de 66,5 anos, que tinham osteoporose e não apresentavam história de câncer de mama ou de endométrio. Foram randomizadas para receber placebo ou 60 ou $120 \mathrm{mg} /$ dia de raloxifeno. Após 40 meses de seguimento observouse redução em $72 \%$ no risco de câncer de mama ${ }^{19}$. Em seguimento ao estudo MORE, outro estudo denominado estudo CORE (Continuing Outcomes relevant to Evista) foi conduzido por quatro anos para avaliar a persistência e ou a maior redução na incidência do câncer de mama $\mathrm{a}^{20}$. As pacientes do estudo MORE que tinham recebido raloxifeno e consentiram participar do estudo foram randomizadas para o uso de raloxifeno $60 \mathrm{mg} / \mathrm{dia}$ ou placebo. Durante esse estudo, as usuárias de raloxifeno apresentaram diminuição de $59 \%(R R=0,4 I ; 95 \%$ de $I C=0,24-0,7 I)$ na incidência de câncer de mama invasor e diminuição de $66 \%(R R=0,34 ; 95 \%$ de $\mathrm{Cl}=0,18-0,66)$ de câncer de mama invasor RE-positivo, quando comparadas com o grupo placebo. $\mathrm{Na}$ análise dos dois estudos em conjunto, nesse período de oito anos, a incidência de câncer de mama invasor foi reduzida em $66 \%$ ( $R R=0,34 ; 95 \%$ de IC = 0,22 - 0,50) e, para os casos RE-positivos, em 76\% (RR=0,24; $95 \%$ de $I C=0,15-0,40$ ), em relação ao grupo placebo. Não foi observada proteção frente aos cânceres não invasivos $(R R=1,78$; $95 \%$ de $I C=0,37-8,6 \mid)^{20}$.

\section{Tamoxifeno versus raloxifeno}

Os resultados do estudo MORE levaram o NSABP a idealizar o estudo STAR (Study of Tamoxifen and Raloxifen)21, em que 19.747 mulheres pós-menopáusicas, com risco de Gail maior que I,66\% em cinco anos ou história de carcinoma lobular in situ, foram randomizadas para receber $20 \mathrm{mg} / \mathrm{dia}$ de tamoxifeno ou $60 \mathrm{mg} / \mathrm{dia}$ de raloxifeno por um período de cinco anos. Os resultados desse estudo mostraram que esse fármaco apresenta a mesma eficácia que o tamoxifeno na quimioprevenção do câncer de mama invasor, ambos apresentando diminuição de risco de 50\% ( $R R=1,02 ; 95 \%$ de $I C=0,82-1,28)$; todavia, como observado no estudo CORE, o raloxifeno não apresentou proteção contra os cânceres não invasores, enquanto que o tamoxifeno diminuiu sua incidência pela metade. Quando da comparação dos dois grupos, observou-se incidência de 1,51 versus 2, I I por 1.000 $(R R=1,40 ; 95 \%$ de $I C=0,98-2,00)$ com $p=0,052$. Na análise dos efeitos colaterais, observou-se, no grupo tratado com raloxifeno, $29 \%$ a menos de trombose venosa profunda e embolia pulmonar ( $R R=0,70 ; 95 \%$ de IC = 0,54-0,9I); e 36\% a menos de câncer de endométrio ( $R R=0,62 ; 95 \%$ de $I C=0,35-1,08)$. Os dois grupos apresentaram a mesma incidência de acidentes vasculares celebrais, ataques cardíacos e fraturas. Observou-se uma menor incidência de catarata $(R R=0,79 ; 95 \%$ de $I C=0,68-0,92$ ) e cirurgias por catarata ( $R R=0,82 ; 95 \%$ de $I C=0,68-0,99)$ no grupo de usuárias de raloxifeno. Estes dados embasam o uso do raloxifeno na quimioprevenção do câncer de mama.

\section{Inibidores da aromatase}

Antes da menopausa, os estrógenos são sintetizados, em grande parte, nos ovários. Assim como outros esteróides sexuais, os estrogênios têm origem na molécula do colesterol, que sofre ação de diversas enzimas presentes em tecidos como gônadas, adipócitos e córtex supra-renal. Após a menopausa, caracterizada a insuficiência ovariana, o córtex supra-renal passa a ser a principal fonte de esteróides sexuais, produzidos na forma de androgênios. Esses androgênios em tecidos periféricos são convertidos em estrogênios pela aromatase, também conhecida como estrógeno sintetase ${ }^{22}$.

Aromatase é uma enzima da família do citocromo P-450 que catalisa a conversão de androgênios em estrogênios agindo, assim, como controladora da taxa de biossíntese de estrogênios. A fonte predominante de aromatase em mulheres pós-menopáusicas é, principalmente, o tecido periférico, especialmente o adiposo. Todavia, também está presente no interior das células do carcinoma mamário e acredita-se ser responsável por incremento na taxa de proliferação deste tumor?.

Já foi demonstrado que as concentrações de estradiol no câncer mamário podem ser até 20 vezes maiores que aquelas observadas no plasma, o que reflete uma grande atividade da aromatase nesta neoplasia, favorecendo a ação predominantemente intrácrina e parácrina do estradiol, corroborando com a indução e promoção da doença23,24,25.

Os inibidores de aromatase determinam, dessa forma, acentuada diminuição nas concentrações de estrogênio, que desempenha papel de destaque no cenário da prevenção do câncer mamário. O seu uso deve ser restrito a pacientes pós-menopáusicas pois, quando do uso no 
menacma, determina diminuição transitória dos estrogênios, o que irá determinar feedback positivo sobre a secreção de GnRH e, conseqüentemente, das gonadotrofinas, determinando estímulo ovariano com aumento das concentrações séricas de estradiol. Este mecanismo pode propiciar o desenvolvimento e crescimento tumoral.

Estudo denominado ATAC (Arimidex, Tamoxifen Alone or in Combination) demonstrou que o uso do anastrazol - inibidor da aromatase - no tratamento adjuvante do câncer inicial de mama proporcionou diminuição tanto na recidiva loco-regional quanto na incidência dessa neoplasia na mama contralateral. Nesse ensaio, que comparou o uso do anastrazol e do tamoxifeno no tratamento adjuvante do câncer de mama, constatou-se redução de 59\% na incidência do câncer de mama contralateral após cinco anos de seguimento no grupo tratado com anastrazol. No grupo tratado com tamoxifeno, observamos maior incidência de trombose venosa profunda, isquemia cerebral, câncer de endométrio, sangramento vaginal e ondas de calor; já nas usuárias de anastrazol, os efeitos adversos mais freqüentes foram fraturas e complicações musculoesqueléticas ${ }^{26}$.

Estudo clínico aleatório foi iniciado para avaliar a eficácia desses agentes frente à prevenção primária do câncer de mama. O estudo IBIS-2 está em fase de recrutamento e irá comparar a eficácia do tamoxifeno e do anastrozol na prevenção do câncer de mama; os primeiros resultados são esperados apenas para 2008.

Outros inibidores de aromatase estão sendo avaliados quanto ao seu potencial na prevenção do câncer de mama; neste sentido, o estudo ApreS (Aromasin Prevention Trial) avaliará randomicamente pacientes portadoras de mutações nos genes BRCA I ou 2 para receberem examestane $25 \mathrm{mg}$ por dia por cinco anos ou placebo; outro estudo, O MA 27, incluirá randomicamente mulheres pósmenopáusicas, com diagnóstico pregresso de CDIS RE-positivo, que receberão anastrazol I mg ou examestane $25 \mathrm{mg} /$ dia, durante cinco anos, associado ao celecoxibe $200 \mathrm{mg} /$ dia por três anos ou ao placebo.

\section{Fitoestrogênios}

Os fitoestrogênios são fenóis que apresentam ação estrogênica, por meio de ligação ao RE e alguma ação antioxidante, que pode impedir a iniciação carcinogênica. Destacam-se, entre esses, as isoflavonas (daidzeína e genisteína) e os flavanóides. Muitos são os mecanismos pelos quais os fitoestrogênios podem desempenhar ação quimiopreventiva, incluindo propriedades antiproliferativas; modulação de enzimas envolvidas na esteroidogênese e proteínas de ligação; indução da apoptose; e provável ação antiangiogênica ${ }^{27}$.

Já foi demonstrado que, na ausência de estrogênio, os fitoestrogênios apresentam efeito estrogênico fraco, ao passo que na presença de estrogênio, eles podem exercer ações antagônicas, sugerindo mecanismo competitivo pelo RE. A genisteína tem mostrado ação supressora sobre a síntese estrogênica por ação inibitória sobre as enzimas aromatase e 17ß-hidroxiesteróide desidrogenase tipo II. Outros prováveis mecanismos de ação da genisteína seria a inibição de DNA topoisomerases; inibição da expressão de fatores transcripcionais do DNA (c-fos e c-jun); e potencialização da ação do fator de crescimento transformacional beta (TFG-B), inibindo a passagem da fase $G_{1}-$ $S$ do ciclo celular, diminuindo a proliferação celular.
Vários estudos estão sendo conduzidos com o intuito de avaliar o potencial quimiopreventivo dos fitoestrogênios; todavia não há nenhum prospectivo, até o momento, que pudesse justificar a habilidade dos fitoestrogênios de diminuir o risco de câncer de mama. Em estudo caso-controle, onde foram avaliados 1.326 casos de câncer de mama e 1.657 controles, foram encontrados os seguintes odds ratio (OR): genisteina $(O R=1,0 ; 95 \%$ de $I C=0,996-1,00 I)$; daidzeina $(\mathrm{OR}=1,00 ; 95 \%$ de $\mathrm{IC}=0,995-\mathrm{I}, 00 \mathrm{I})$; e para as isoflavonas totais $(\mathrm{OR}=0,99 ; 95 \%$ de $\mathrm{IC}=0,98-1,0 \mathrm{I})$, que demostram nenhum impacto sobre 0 câncer de mama nas doses habituai $5^{28}$.

\section{Análogos de GnRH}

O uso dos análogos objetiva suprimir temporariamente a função ovariana, gerando um status pós-menopausal e, dessa forma, reduzindo a exposição cumulativa aos hormônios esteróides ovarianos.

Os análogos de GnRH já demonstraram sua eficácia para o tratamento do câncer de mama em pacientes pré-menopáusicas que apresentam tumores positivos para RE. Todavia, não existem dados que embasem o seu uso na prevenção do câncer de mama. No momento, quatro ensaios clínicos, fase II, desenhados para avaliar a eficácia dos análogos na prevenção do câncer de mama em pacientes de alto risco, encontram-se em fase de recrutamento. Um conduzido pelo Nacional Cancer Institute (NCl), em que as pacientes receberão deslorelina, estradiol e testosterona por meio de spray nasal uma vez ao dia, por seis a dez meses; e outros três, em que a goserelina será associada com substâncias antiosteoporóticas: raloxifeno (RAZOR), tibolona (TIZER) e com ibandronato de sódio (GISS). Entretanto, os resultados ainda tardarão.

\section{Inibidores da ciclooxigenase-2}

As prostaglandinas (PG) são mediadores lipídicos de processos fisiológicos normais, incluindo agregação plaquetária, funções gástrica e renal, reprodução, processos inflamatórios e manutenção da integridade e tônus vascular. A maioria das células tem a capacidade de sintetizálas, sendo o primeiro passo a hidrólise de fosfolípides para produzir ácido aracdônico livre, por meio da ação catalizadora da fosfolipase $A_{2}$. Na seqüência desta via metabólica, a depender da ação catalizadora da ciclooxigenase, que insere uma molécula de oxigênio no ácido aracdônico, ocorre a produção da PGG ; esta é rapidamente convertida em $\mathrm{PGH}_{2}$, pela ação peroxidativa da $\mathrm{COX}$, que, por sua vez, sob efeito das isomerases tecíduo-específicas, transformam-se em outras PG ( $\left.P_{G E}, P_{2} D_{2}, P G F_{2} a, P G I_{2}\right)$, assim como em tromboxano e prostaciclinas $^{29}$.

Existem duas isoenzimas ciclooxigenase: COX-I e COX-2; a primeira é constitutiva e expressa pela maioria dos tecidos e parece ser responsável pela produção das PGs que modulam funções fisiológicas ${ }^{30}$. Em contraste, a segunda não é detectável na maioria dos tecidos normais e é induzida por citoquinas, fatores de crescimento, oncogenes e promotores tumorais nos quadros inflamatórios e em tecidos tumorais, contribui para a síntese de $\mathrm{PG} s^{3 !}$.

A COX-2 parece atuar na carcinogênese de várias formas, incluindo efeitos sobre a apoptose, proliferação celular, imunomodulação, agressão do tumor, neoangiogênese e invasão tumoral. A prostaglandina $E_{2}$ parece ser a mediadora destes efeitos, particularmente $d a$ apoptose e neoangiogênese ${ }^{29}$. 
Em estudo prospectivo para avaliar o risco de câncer de mama em usuárias de aspirina, Egan et al. ${ }^{32}$ avaliaram os dados do Nurses 'Health Study, em que 2.4I4 casos de câncer mamário foram diagnosticados. Os autores encontraram RR de 1,0, após 12 anos de seguimento, no grupo das pacientes que faziam uso regular de aspirina (duas a três vezes por semana), mostrando que esta não reduziu o risco de câncer de mama.

Johnson et al. ${ }^{33}$, em estudo de coorte com 27.616 mulheres na pós-menopausa, identificaram 938 casos de câncer de mama em seis anos de seguimento, traduzindo RR de 0,7 I (95\% de IC= $0,58-0,87)$ para aquelas que fizeram uso de aspirina seis vezes ou mais por semana, quando comparadas com as que não usaram esta medicação.

Conduzindo estudo de coorte, Harris et al. ${ }^{34}$, após cinco anos de seguimento, encontraram 393 casos de câncer de mama em 32.505 pacientes, constatando decréscimo de $50 \%$ na incidência de câncer de mama nas usuárias regulares de ibuprofeno $(p<0,01)$ e de $40 \%$ nas que faziam uso regular de aspirina $(p<0,05)$, sugerindo que os antiinflamatórios não hormonais podem ser agentes quimiopreventivos efetivos na profilaxia do câncer de mama.

Recente metanálise envolvendo I 4 estudos que avaliaram o uso de antiinflamatórios não hormonais sobre o risco de câncer de mama confirmou pequena redução no risco (RR combinado de 0,82; $95 \%$ de IC: 0,75-0,89); os dados, entretando, não foram suficientes para avaliar dose, freqüência e duração do uso de qualquer tipo de antiinflamatório não hormonal $\left.\right|^{35}$.

Em estudo caso-controle em que foram avaliados 7.006 casos de câncer de mama, a relação entre o uso de antiinflamatórios não hormonais e o risco de desenvolvimento desta neoplasia foi avaliada. O RR para as pacientes que faziam uso regular de aspirina foi de 0,78 (95\% de IC =0,63-0,97). Os melhores resultados foram observados entre as pacientes pré-menopáusicas com $R R$ de 0,62 . Os autores ainda observaram que tanto a aspirina $(R R=0,86)$ quanto o ibuprofeno $(R R=0,85)$ mostraram resultados semelhantes e o status do RE não influenciou nos resultados observados ${ }^{36}$.

Harris et a. $1^{37}$, em 2006, conduziram o primeiro estudo casocontrole que comparou os inibidores seletivos e não seletivos de COX2 na prevenção do câncer de mama. Foi avaliado um total de 323 casos de câncer de mama incidentais que foram pareados com 649 pacientes que não apresentavam a doença. Os resultados mostraram significante redução de risco no grupo das usuárias de inibidores seletivos de COX-2 (OR =0,29; $95 \%$ de IC =0, I4-0,59), aspirina (OR =0,49; $95 \%$ de $\mathrm{IC}=0,26-0,94)$ e ibuprofeno ou naproxeno $(O R=0,36 ; 95 \%$ de IC $=0,18-0,72)$, sinalizando, assim, importante ação quimioprotetora dos inibidores de COX-2.

\section{Retinóides}

Os retinóides, derivados naturais ou sintéticos da vitamina $A$, desempenham papel fundamental na proliferação e diferenciação celular; podem suprimir a promoção tumoral e modificar determinadas propriedades de células neoplásicas invasoras por meio da ativação ou repressão de genes específicos.
Os receptores dos retinóides estão presentes no epitélio mamário normal e são importantes para o adequado desenvolvimento da glândula mamária. Ainda que os mecanismos envolvidos na inibição da proliferação celular promovida pelos retinóides não sejam completamente conhecidos, evidências experimentais sugerem que múltiplas vias metabólicas de transdução estejam provavelmente envolvidas e que resultem em ações diretas ou indiretas sobre a expressão gênica, propiciando mecanismo regulador sobre processos celulares incluindo proliferação, diferenciação e apoptose celular ${ }^{38}$.

Veronesi et al. ${ }^{39}$, em estudo aleatório com 2.972 pacientes previamente tratadas por câncer de mama, avaliaram o potencial da fenretidina (derivado sintético do ácido retinóico), em prevenir a recidiva ipsilateral e o surgimento de câncer na mama contralateral. Após 97 meses de seguimento, não observaram diferença significativa entre o grupo tratado e o placebo. Quando da estratificação das pacientes em pré e pós-menopáusicas, notou-se tendência para o benefício do uso em pacientes prémenopáusicas $(R R=0,66 ; 95 \%$ de $I C=0,41-1,07$ ), o mesmo não sendo observado nas pacientes pós-menopáusicas ( $R R=$ I,32; $95 \%$ de $I C=0,82-2,15)$.

Uma nova classe de retinóides, denominados rexinóides, a qual apresenta especificidade pelo receptor retinóide $X$, que lhe confere menor toxicidade, encontra-se em estudo para medir sua eficácia na quimioprevenção mamária. Estudos preliminares têm demonstrado que o rexinóide bexaroteno determina diminuição da expressão da COX-2, sendo esse um dos principais mecanismos dessa substância na prevenção mamária. Estudos prospectivos e aleatórios são aguardados para confirmar a ação quimiopreventiva dessas substâncias.

\section{Estatinas}

As estatinas são substâncias que determinam diminuição da síntese de colesterol por meio da inibição da enzima 3-hidroxi3metilglutaril-coenzima A (HMG-CoA) redutase. A propriedade antiproliferativa das estatinas deve estar relacionada à inibição da HMG-CoA redutase, propiciando diminuição nas concentrações do ácido mevalônico e prevenindo a transdução de genes envolvidos na proliferação celular.

Em estudo caso-controle, em que foi investigada a relação das estatinas com o desenvolvimento do câncer de mama, em 40.421 mulheres americanas, observou-se que as usuárias de estatinas apresentaram diminuição de risco de desenvolvimento do câncer mamário em $51 \%(O R=0,49 ; 95 \%$ de IC $=0,38-0,62 ; p<0,000 I)$ quando comparadas às não usuárias ${ }^{40}$.

Recente metanálíse, ao estudar sete estudos aleatórios e nove observacionais, constatou que as estatinas não exibem qualquer potencial quimiopreventivo $(R R=1,03 ; 95 \% \text { de } I C=0,93-|,| 4)^{4 \mid}$.

\section{CONCLUSÃo}

A quimioprevenção do câncer de mama é factível; deve sempre ser realizada com critério e dentro de preceitos rígidos de eleição, utilizando-se medicações comprovadamente eficazes. $O$ tamoxifeno já teve sua eficácia demonstrada em dois estudos aleatórios, NSABPPI e STAR (nível I de evidência); pode ser utilizado em pacientes com diagnóstico prévio de carcinoma lobular in situ ou hiperplasia 
ductal ou lobular com atipias, mulheres acima de 35 e abaixo de 65 anos com risco de Gail superior a 1,66\% para os próximos cinco anos, e em portadoras de mutação gênica no gene BRCA-2, que não tenham optado pela mastectomia profilática, ainda que os estudos não sejam conclusivos quanto a esta indicação. $\mathrm{O}$ uso em associação com outros agentes ou por mais de cinco anos ainda não foi avaliado; dessa forma, deve ser prescrita na dose de 20 mg diárias por um período de cinco anos.

O raloxifeno comprovou sua eficácia por meio do estudo STAR; pode ser prescrito na dose de $60 \mathrm{mg} / \mathrm{dia}$ por cinco anos em pacientes após a menopausa, com diagnóstico de carcinoma lobular in situ ou hiperplasia ductal ou lobular com atipias, ou risco de Gail superior a 1,66\% em cinco anos. Exibe como vantagens ao tamoxifeno taxas menores de fenômenos tromboembólicos e câncer de endométrio, e, entre as desvantagens, não exerce proteção contra o carcinoma ductal in situ.

Os inibidores de aromatase já demonstraram sua eficácia na prevenção do câncer de mama contralateral, todavia, é imperioso aguardar os resultados dos estudos aleatórios (Ibis-II, NSABP-PII e ApreS) para confirmar ou não sua eficácia na prevenção primária.

Estudos do tipo coorte e caso-controle têm demonstrado a eficácia dos antiinflamatórios, em especial os inibidores de COX-2, na quimioprevenção; entretanto, estudos aleatórios são necessários para determinar a melhor substância, dose ideal e período de uso, além de propiciar um melhor nível de evidência (I) para esse grupo de fármacos.

\section{Conflito de interesse: não há.}

\section{SUMMARY}

\section{Current status of breast CANCER CHemoprevention}

Chemoprevention is defined as the use of natural or synthetic chemical agents to reverse, suppress or prevent carcinogenic progression of invasive cancer. Drugs that act as chemoprevention agents for breast cancer are divided into two major groups: drugs that prevent Estrogen Receptor (ER) - positive breast cancers [selective estrogen receptor modulators (SERM), aromatase inhibitors GnKH agonists and phytoestrogens] and drugs that prevent ER - negative breast cancers [cyclooxygenase-2 (COX-2) inhibitors, retinoids, statins, receptor tyrosine, kinase inhibitors, monoclonal antibody against HER2 and telomerase inhibitors]. Results from the NSABP Study of Tamoxifen and Raloxifene (STAR), which compared the risk-reducing efficacy as well as toxicity of these two SERMs in a similar high-risk for breast cancer population, showed that Raloxifene is as effective as Tamoxifen in reducing the risk of non-invasive breast cancer $(p=.83)$. It has a statistically significant lower risk of thromboembolic events and cataracts, however a non- statistically significant higher risk of noninvasive breast cancer. Based on promising data involving reduction of contralateral breast cancer risk in adjuvant studies, several aromatase inhibitors, including letrozole, anastrozole and exemestane, are being included in trials to evaluate their efficacy in breast cancer prevention in both case-control and cohort studies As such randomized studies to confirm this efficacy are needed. Positive results of several recent clinical trials for preventing breast cancer in high-risk populations suggest that chemoprevention is a rational and attractive strategy. [Rev Assoc Med Bras 2006; 52 (6): 453-9]

KEY Words: Breast cancer. Chemoprevention. Tamoxifen. Raloxifene, Aromatase. Cyclooxygenase.

\section{RefERÊNCIAS}

I. Johnson-Thompson MC, Guthrie J. Ongoing research to identify environmental risk factors in breast carcinoma. Cancer. 2000;88: I 224-9.

2. Feuer EJ, Wun L, Boring CC, Flaunders WD, Timmel MJ, Tong T. The life time risk of developing breast cancer. J Natl Cancer Inst. 1993;85:892-97.

3. Brasil. Ministério da Saúde INCA/Comprev. Estimativa 2006: Incidência de câncer no Brasil. Rio de Janeiro; 2005.

4. Bilimoria MM, Morrow M. The woman at increased risk for breast cancer: evaluation and management strategies. Cancer J Clin. 1995;45:263-78.

5. Badawi AF, Badr MZ. Chemoprevention of breast cancer by targeting cyclooxygenase-2 and peroxisome proliferator-activated receptor-g. Int J Oncol. 2002;20: I 109-22.

6. Molina LA, Dalben I, Luca LA. Análise das oportunidades de diagnóstico precoce para as neoplasias malignas de mama. Rev Assoc Med Bras. 2003;49: I 85-90.

7. Prichard RS, Hill ADK, Dijkstra B, Mcdermott EW, O'higgins NJ. The chemoprevention of breast cancer. British J Surg. 2003;90:772-83.

8. Sporn MB. Approaches to prevention of the epithelial cancer during the pre-neoplastic period. Cancer Res. 1976;36:2699-702.

9. Cuzick J, Baum M. Tamoxifen and contralateral breast cancer. Lancet. 1985;2:282-4.

10. Fisher B, Dignam J, Bryant J, DeCillis A, Wickerham DL, Wolmark N, et al. Five versus more than 5 years of tamoxifen for breast cancer patients with negative lymph nodes and estrogen receptor-positive tumors. J Natl Cancer Inst. 1996;88: I 529-42.

II. Fisher B, Costantino JP, Wickerham DL, Redmond CK, Kavanah M, Cronin WM. Tamoxifen for prevention of breast cancer: report of the National Surgical Adjuvant Breast and Bowel Project P- I Study. J Natl Cancer Inst. 1998;90: |37|-88.

12. Powles T, Eeles R, Ashley SE, Easton D, Chang J, Dowsett L. Interim analysis of the incidence of breast cancer in the Royal Marsden Hospital tamoxifen randomized chemoprevention trial. Lancet. 1998;352:98- I 0 I .

13. Veronesi U, Maisonneuve P, Sacchini V, Rotmensz N, Boyle P. Tamoxifen for breast cancer among hysterectomised women. Lancet. 2002; 359: I | 22-24.

14. Investigators. First results from the International Breast Cancer Intervention Study (IBIS-I): a randomised prevention trial. Lancet. 2002;360:817-24

15. Gail MH, Brinton LA, Byar DP. Projecting individualized probabilities of developing breast cancer for white females who are being examined annually. J Natl Cancer Inst. 1989;81: | 879-86.

16. Fisher B, Constantino JP, Wickerham: J Natl Cancer Inst. 2005;97: I 6521662.

17. Cuzick J, Powles T, Verones U, Forbes J, Edwards R, Ashley, et al. Overview of the main outcomes in breast-cancer prevention trials. Lancet. 2003;361:296-300.

18. Jordan, VC, Morrow M. Tamoxifen, raloxifen, and the prevention of breast cancer. Endocr Rev. 1999;20:253-78.

19. Cauley JA, Norton L, Lippman ME, Eckert S, Krueger KA, Purdie Dw. Continued breast cancer risk reduction in postmenopausal women treated with raloxifen. Breast Cancer Res Treat. 200 I;65: I 25-34.

20. Martino S, Cauley JA, Barrett-Connor E, Powles TJ, Mershon J, Disch D, et al. Continuing outcomes relevant to Evista: breast cancer incidence in postmenopausal osteoporotic women in a randomized trial of raloxifene.J Natl Cancer Inst. 2004;96: I75 I-6I. 
21. Vogel V, Constantino JP, Wickerham DL, Cronin WM, Cecchini RS, Atkins $\mathrm{JN}$. Effects of tamoxifen vs raloxifene on the risk of developing invasive breast cancer and other disease outcomes. the NSABP Study of Tamoxifen and Raloxifene (STAR) P-2 trial. JAMA. 2006;295:2727-4I.

22. Purohit A, Newman SP, Reed MJ. The role of cytoquines in regulating estrogen synthesis. implications for the etiology of breast cancer. Breast Cancer Res. 2002;4:65-69.

23. Pasqualini, JR, Chetrite G, Blacker C, Feinstein MC, Delalonde L, Talb I M, et al. Concentrations of estrone, estradiol, and estronesulfate and evaluation of sulfatase and aromatase activities in pre- and postmenopausal breast cancer patients. J Clin Endocrinol Metab. 1996;81: | 460-4.

24. Sun X.Z., Zhou D; Chen S. Autocrine and paracrine actions of breast tumor aromatase. A three-dimensional cell culture study involving aromatase transfected MCF-7 andT-47D cells. J Steroid Biochem Mol Biol. 1997;63:29-36.

25. Castagnetta LA, Lo Casto M, Granata OM, Polito L, Calabro M, Lo BA, et al. Estrogen content and metabolism in human breast tumor tissue and cells. Ann NY Acad Sci. 1996;784:3 | 4-24.

26. ATAC trialists' Group. Results of the ATAC (Arimidex, Tamoxifen, Alone or in Combination) trial after completion of 5 years' adjuvant treatment for breast cancer. Lancet. 2005;365:60-2.

27. Wagner JD, Anthony MS, Cline JM. Soy phytoestrogens: research on benefits and risks. Clin Obstet Gynecol. 2001;44:843-52.

28. Horn-Ross P, John E, Lee M.. Phytoestrogen consumption and breast cancer risk in a multiethnic population. Am J Epidemiol. 200 I ; $54: 434$ 41

29. Hawk ET, Viner JL, Asad Umar A, William F, Anderson WF, Sigman CC, et al. Development of COX inhibitors in cancer prevention and therapy. Am J Cancer. 2003;2:27-55

30. Dempke W, Rie C, Grothey A, Schmoll H. Cyclooxygenase-2: a novel target for cancer chemotherapy? J Cancer Res Clin Oncol. 200 I ; I 27:4 I I-7.

31 . Fosslien E. Molecular pathology of cyclooxygenase-2 in neoplasia. Ann Clin Lab Sci. 2000;30:3-21.
32. Egan KM, Stampfer MJ, Giovannucci E, Rosner BA, Colditz GA. Prospective study of regular aspirin use and the risk of breast cancer. J Natl Cancer Inst. 1996;88:988-93.

33. Johnson TW, Anderson KE, Lazovich D, Folsom AR. Association of aspirin and nonsteroidal anti-inflammatory drug use with breast cancer. Cancer Epidemiol Biomarkers Prev. 2002; I | | 586-9| .

34. Harris RE, Kasbari S, Farrar WB. Prospective study of nonsteroidal antiinflammatory drugs and breast cancer. Oncol Rep. 1999;6:71-3.

35. Khuder SA, Mutgi AB. Breast cancer and NSAID use: a meta-analysis. Br | Cancer. 2001;84: I | 88-92.

36. Zhang Z, Yamshita H, Toyama T, Hara Y, Omoto Y, Sugiura H, et al. Semiquantitative immunohistochemical analysis of aromatase expression in cuctal carcinoma of the breast. Breast Cancer Res Treat. 2002;74: 47-53.

37. Harris RE, Beebe-Donk J, Alshafie A. Reduction in the risk of human breast cancer by selective cyclooxygenase-2 (COX-2) inhibitors. BMC Cancer. 2006;6:27.

38. Simeone AM, Tari AM How retinoids regulate breast cancer cell proliferation and apoptosis. Cell Mol Life Sci. 2004;6 I : | 475-84.

39. Veronesi U, Palo G, Marubini E, Costa A, Formelli F, Mariani L, et al. Randomized Trial of Fenretinide to Prevent Second Breast Malignancy in Women With Early Breast Cancer. I Natl Cancer Inst. 1999;91: I 847-56.

40. Kochhar R, Khurana V, Bejjanki H. Statins reduce breast cancer risk: A case control study in US female veterans. J Clin Oncol. 2005;23:7S.

4I. Bonovas S, Filioussi K, Tsavaris N, Sitaras NM. Use of statins and breast cancer: a meta-analysis of seven randomized clinical trials and nine observational studies. J Clin Oncol. 2005;23:8606- 12.

Artigo recebido: 03/07/06

Aceito para publicação: 04/07/06 